\title{
The forkhead transcription factor FOXO1 mediates cisplatin resistance in gastric cancer cells by activating phosphoinositide 3-kinase/Akt pathway
}

\author{
Jinju Park • Young San Ko · Jiyeon Yoon • Min A. Kim • \\ Jong-Wan Park · Woo Ho Kim • Youngsun Choi • \\ Ji Hun Kim · Younghee Cheon · Byung Lan Lee
}

Received: 5 July 2013/Accepted: 21 October 2013/Published online: 8 November 2013

(C) The International Gastric Cancer Association and The Japanese Gastric Cancer Association 2013

\begin{abstract}
Background Cisplatin (CDDP) is one of the most important chemotherapeutic agents in the treatment of advanced gastric cancer, but its efficacy is limited by CDDP resistance. Because the transcription factor FOXO1 is related to chemoresistance in various cancer cells, we investigated the function of FOXO1 in CDDP resistance in human gastric cancer cells.

Methods Human gastric cancer cell lines MKN45 and SNU-601 were used. FOXO1 activation was modulated by transfection of FOXO1 AAA mutant gene or FOXO1 shRNA. The effects of FOXO1 on cell growth and CDDP cytotoxicity were assessed by crystal violet assay. Protein
\end{abstract}

J. Park · W. H. Kim - B. L. Lee

Department of Tumor Biology, Cancer Research Institute, Seoul

National University College of Medicine, Seoul, Korea

Y. S. Ko · J. Yoon · Y. Choi · B. L. Lee $(\bowtie)$

Department of Anatomy, Seoul National University College of Medicine, 103 Daehak-ro, Jongno-gu, Seoul 110-799, Korea

e-mail: dslanat@snu.ac.kr

M. A. Kim - W. H. Kim

Department of Pathology, Seoul National University College of Medicine, Seoul, Korea

J.-W. Park · B. L. Lee

Ischemic/Hypoxic Disease Institute Medical Research Center, Seoul National University College of Medicine,

Seoul 110-799, Korea

J. H. Kim

Department of Pathology, Asan Medical Center, Ulsan

University College of Medicine, Seoul, Korea

Y. Cheon

Department of Pediatrics, Yeungnam University College of Medicine, Daegu 705-703, Korea expressions of FOXO1, p110 $\alpha$, pAkt, and Akt were analyzed by Western blotting, and FOXO1 mRNA expression was evaluated by semiquantitative reverse transcription-polymerase chain reaction. FOXO1 activity was determined by luciferase reporter assay, and cell apoptosis was assessed by DAPI staining and Western blotting for PARP cleavage.

Results Cisplatin treatment induced FOXO1 expression and activation in both gastric cancer cell lines. FOXO1 overexpression increased the CDDP resistance without changes in cell growth, whereas FOXO1 silencing enhanced CDDP cytotoxicity along with apoptotic characteristics. Both constitutive and CDDP-induced FOXO1 activations were accompanied by an increase in $\mathrm{p} 110 \alpha$ and pAkt expression. Furthermore, Akt inhibition by LY294002 treatment restored the CDDP cytotoxicity that was suppressed by FOXO1 overexpression.

Conclusion FOXO1 inhibits CDDP-induced apoptosis in gastric cancer cells via activating PI3K/Akt pathway. Thus, FOXO1 may be an useful pharmacological indicator to predict CDDP efficacy in gastric cancer treatment.

Keywords FOXO1 · Gastric cancer - Cisplatin resistance $\cdot \mathrm{PI} 3 \mathrm{~K} / \mathrm{Akt}$

\section{Introduction}

Gastric cancer is one of the most common cancers and a major cause of cancer-related death worldwide [1]. However, the cure rate of this disease is limited because of the ineffectiveness of chemotherapy and radiotherapy. Thus, evaluation of the chemosensitivity of gastric cancer cells to anti-cancer agents based on phenotypic difference of individual cell lines will provide more information for choosing the correct drugs for gastric cancer patients. 
Cisplatin (cis-diamminedichloroplatinium (II), CDDP) is a front-line chemotherapeutic agent in the treatment of advanced gastric cancer [2]. However, almost all cancer cells acquire resistance to CDDP, which reduces its efficacy [3]. Thus, molecules and factors that are capable of predicting responses of gastric cancer patients to CDDP treatment require investigation.

The FOXO (Forkhead box, class O) is a subfamily of forkhead transcription factor and consists of FOXO1, FOXO3A, FOXO4, and FOXO6 [4]. FOXO transcription factors are involved in diverse intracellular signaling pathways and regulate cell-cycle arrest, apoptosis, DNA damage repair, and detoxification of reactive oxygen species by regulating specific gene settings [5, 6]. The FOXO signaling is regulated by interactions with other intracellular proteins as well as their posttranslational modifications such as phosphorylation [7].

The function of FOXO1 in chemoresistance in cancer cells has been evaluated in in vitro studies [8-10]. FOXO1 increased doxorubicin resistance in breast cancer cells [8] and paclitaxel resistance in ovarian cancer cells [9], whereas it decreased CDDP resistance in ovarian cancer cells [10]. Thus, the effect of FOXO1 on the chemoresistance of cancer cells may differ according to the drug and cell type investigated.

The serine-threonine kinase Akt/protein kinase B is a well-known cell survival signal that contributes chemoresistance in a variety of cancer cells [11]. A recent study [12] has demonstrated that Akt overexpression decreases the chemosensitivity of gastric cancer cells to CDDP in vitro and in vivo. Although FOXO1 is a downstream target of Akt in human cancers [13], association between FOXO1 activation and chemoresistance in gastric cancer cells has not been reported.

In the present study, we investigated whether FOXO1 activation is related to CDDP resistance in gastric cancer cell lines. For this, we modulated FOXO1 activation by transfection of FOXO1 AAA mutant gene or FOXO1 shRNA. In addition, we examined the association between FOXO1 and phosphoinositide-3-kinase (PI3K)/Akt pathway in relation to CDDP resistance in gastric cancer cells.

\section{Materials and methods}

\section{Chemicals}

Cisplatin was purchased from Sigma (St. Louis, MO, USA), and LY294002 was purchased from Cell Signaling Technology (Beverly, MA, USA).

Cell lines and cultures

Human gastric cancer cell lines MKN45 and SNU-601 were purchased from the Korean Cell Line Bank (Seoul,
Korea). Cells were cultured in RPMI1640 (Life Technologies, Grand Island, NY, USA) supplemented with $10 \%$ fetal bovine serum (FBS), $2 \mathrm{mg} / \mathrm{ml}$ sodium bicarbonate, $100 \mathrm{U} / \mathrm{ml}$ penicillin, and $100 \mu \mathrm{g} / \mathrm{ml}$ streptomycin (Life Technologies) at $37{ }^{\circ} \mathrm{C}$ in a humidified $95 \%$ air and $5 \%$ $\mathrm{CO}_{2}$ atmosphere.

Stable transfection with a plasmid expressing FOXO1 AAA mutant gene

An expression vector, pcDNA3, with a human FOXO1 AAA mutant gene (Addgene plasmid 13508), was purchased from Addgene (Cambridge, MA, USA). This vector encodes a constitutively active FOXO1 containing a threonine-to-alanine substitution at residue 24 and serine-toalanine substitution at 256 and 319 . This construct $(1 \mu \mathrm{g})$ or empty pcDNA3 vector $(1 \mu \mathrm{g})$ was transfected into $3 \times 10^{5}$ cells/well in six-well plates using LipofectAMINE Plus (Life Technologies) according to the manufacturer's instructions. Pooled G418 (3 $\mu \mathrm{g} / \mathrm{ml})$-resistant cells were used for further analysis.

\section{Lentivirus-mediated shRNA silencing of FOXO1}

FOXO1 shRNA lentiviral particles and nontargeting shRNA control particles were purchased from Sigma. The sequence of the shRNA targeting FOXO1 used in the present study is the following: CCGGGCCTGTTATC AATCTGCTAAACTCGAGTTTAGCAGATTGATAACA GGCTTTTTG. The nontargeting shRNA control particles contain four base-pair mismatches within the short hairpin sequence to any known human or mouse gene. The viral infection was performed by incubating gastric cancer cells in the culture medium containing lentiviral particles for $12 \mathrm{~h}$ in the presence of $5 \mu \mathrm{g} / \mathrm{ml}$ Polybrene (Santa Cruz Biotechnology, Santa Cruz, CA, USA). Pooled puromycin $(2 \mu \mathrm{g} / \mathrm{ml})$-resistant cells were harvested and stored for further analysis.

\section{Western blotting}

Cell lysates were prepared in 100-200 $\mu \mathrm{l} 1 \times$ sodium dodecyl sulfate (SDS) lysis buffer [125 mM Tris- $\mathrm{HCl}(\mathrm{pH}$ 6.8), $4 \%$ SDS, $0.004 \%$ bromophenol blue, and $20 \%$ glycerol]. Protein contents were measured using BCA Protein Assay Reagent (Pierce, Rockford, IL, USA). Equal amounts of proteins were loaded onto a $10 \%$ discontinuous SDS/polyacrylamide gel and electrophoretically transferred to PVDF membranes (Millipore, Billerica, MA, USA) blocked with $5 \%$ nonfat dry milk in phosphate-buffered saline-Tween-20 $(0.1 \%, \mathrm{v} / \mathrm{v})$ for $1 \mathrm{~h}$. The membranes were then incubated at $4{ }^{\circ} \mathrm{C}$ overnight with or without $2 \mathrm{~h}$ incubation at room temperature with one of the following 
primary antibodies: rabbit anti-FOXO1 (1:1000, Cell Signaling Technology), rabbit anti-poly(ADP ribose)polymerase (PARP) (1:1000, Cell Signaling Technology), rabbit anti-p110 $\alpha$ (1:1000, Cell Signaling Technology), rabbit anti-phospho-Akt ${ }^{\mathrm{Ser} 473}(1: 1000$, Cell Signaling Technology), rabbit anti-Akt (1:1000, Cell Signaling Technology), and mouse anti- $\beta$-actin (1:1000, Santa Cruz Biotechnology). Horseradish peroxidase-conjugated anti-rabbit IgG (1:2000, Zymed, San Francisco, CA, USA) or anti-mouse IgG (1:2500, Santa Cruz Biotechnology) was used as a secondary antibody. Enhanced chemiluminescence was used to detect the immunoreactive proteins. Equal protein loading was confirmed by $\beta$-actin.

Semiquantitative reverse transcription-polymerase chain reaction (SQ RT-PCR)

Semiquantitative reverse transcription-polymerase chain reaction was performed to determine the transcript level of FOXO1 in human gastric cancer cells, and the amplification of $\beta$-actin transcripts was used as the control to normalize the transcript levels of FOXO1. Total RNAs were isolated using TRIZOL reagent (Invitrogen, Carlsbad, CA, USA), and reverse transcription was performed to synthesize cDNAs in a $20-\mu 1$ reaction mixture containing each gene-specific primer, $1 \mu \mathrm{g}$ RNA, $2 \times$ reaction buffer, $0.4 \mu \mathrm{l}$ Taq polymerase, and $1.2 \mathrm{mM} \mathrm{MgCl}_{2}$. The cDNAs of FOXO1 transcripts were all amplified for 25 cycles (30 s at $94{ }^{\circ} \mathrm{C}, 1 \mathrm{~min}$ at $57^{\circ} \mathrm{C}$, and $1 \mathrm{~min}$ at $72{ }^{\circ} \mathrm{C}$ ), and the cDNAs of $\beta$-actin transcripts were amplified for 18 cycles $\left(94{ }^{\circ} \mathrm{C}\right.$ for $30 \mathrm{~s}, 52{ }^{\circ} \mathrm{C}$ for $30 \mathrm{~s}$, and $70{ }^{\circ} \mathrm{C}$ for $30 \mathrm{~s}$ ). The PCR cycling numbers had been optimized to avoid the amplification saturation. Then, $5 \mu \mathrm{l}$ of RT-PCR product was separated on $1 \%$ agarose gels, which were subsequently stained with ethidium bromide. Primer sequences were $5^{\prime}$-GCAGATCTACGAGTGGATGGTC- $3^{\prime}$ and $5^{\prime}$-A AACTGTGATCCAGGGCTGTC- $3^{\prime}$ for FOXO1, and $5^{\prime}$-A CACCTTCTACAATGAGCTG-3' and 5'-CATGATGG AGTTGAAGGTAG- $3^{\prime}$ for $\beta$-actin.

\section{Luciferase reporter assay}

Gastric cancer cells were seeded in 24-well plates at a density of $3 \times 10^{4}$ cells/well and were transiently cotransfected with $0.4 \mu \mathrm{g}$ forkhead responsive element (FHRE)-luciferase reporter plasmid (reporter construct in which a small region of the Fas ligand promoter containing the three FHREs, Addgene plasmid 1789) and $0.4 \mu \mathrm{g} \beta$ galactosidase vector, an internal control, using Lipofectamine Plus (Life Technologies). Twenty-four hours after transfection, assays for luciferase and $\beta$-galactosidase were carried out using a Dual-Luciferase Reporter Assay System (Promega, Madison, WI, USA). Luciferase activity was measured on an AutoLumat LB 9505c luminometer (Berthold Analytical Instruments, Nashua, Germany) and was normalized by $\beta$-galactosidase activity. Luciferase activity in control cells was arbitrarily set to 1 .

\section{Cell proliferation and cytotoxicity assay}

Cells were seeded onto 24-well plates, at a density of $1 \times 10^{4}$ cells/well for cell growth, and cultured for 4 days. To study the effects of CDDP on cell viability, $3 \times 10^{4}$ cells/well were seeded onto 24 -well plates. After $24 \mathrm{~h}$, cells were treated with various concentrations of CDDP dissolved in $0.02 \%$ dimethyl sulfoxide (DMSO) (range, $0.1-3 \mu \mathrm{g} / \mathrm{ml}$ ) for $24 \mathrm{~h}$. For PI3K/Akt inhibition, cells were treated with $20 \mu \mathrm{M}$ LY294002. Control columns contained cells without drug and blank columns contained medium alone. Cell numbers were measured indirectly using the method reported by Kim et al. [14]. Briefly, attached cells were stained with $0.2 \%$ crystal violet aqueous solution in $20 \%$ methanol for $10 \mathrm{~min}$, dissolved in $10 \%$ SDS, transferred into 96-well plates, and the absorbance was measured at $570 \mathrm{~nm}$ using an enzyme-linked immunosorbent assay reader (Bio-Rad, Hercules, CA, USA). Absorbance values were normalized to the values obtained for the medium control group cells to determine the survival percentage.

\section{4'-6-Diamidino-2-phenylindole (DAPI) staining}

Apoptosis was evaluated by DAPI staining as described previously [15]. Briefly, cells were fixed with $4 \%$ paraformaldehyde for $30 \mathrm{~min}$, washed three times with phosphatebuffered saline (PBS), and stained with DAPI $(1 \mu \mathrm{g} / \mathrm{ml})$ in the dark for $30 \mathrm{~min}$ and then examined under a fluorescence microscope. Cells were considered apoptotic if their nuclei were condensed or fragmented.

Statistical analysis

Data were analyzed by one-way analysis of variance (ANOVA), and differences were considered significant at $P<0.05$ in the Newman-Keuls multiple-comparisons test. GraphPad Prism 4.00 for Windows Vista (GraphPad Software, San Diego, CA, USA) was used to conduct the analysis.

\section{Results}

CDDP induces FOXO1 expression and activation in gastric cancer cells

We investigated the correlation between FOXO1 and chemoresistance to CDDP in gastric cancer cells. First, we 
treated parent gastric cancer cell lines MKN45 and SNU601 with CDDP $(3 \mu \mathrm{g} / \mathrm{ml})$ for $24 \mathrm{~h}$ and found that the expressions of FOXO1 protein (Fig. 1a, top) and mRNA (Fig. 1a, bottom) were increased in both cell lines. The induction of FOXO1 activation was confirmed by a luciferase reporter assay with a FHRE luciferase plasmid (Fig. 1b). These results indicate that CDDP activates FOXO1 in gastric cancer cells.

FOXO1 activation in gastric cancer cell lines confers resistance to CDDP-induced cytotoxicity

To clarify the role of FOXO1 in CDDP resistance in gastric cancer cells, we modulated the FOXO1 activation. As Gao et al. [10] reported that FOXO1 increased CDDP cytotoxicity of ovarian cancer cells, we transfected the FOXO1 AAA mutant gene, a constitutively active FOXO1 mutant, into gastric cancer cell lines MKN45 and SNU-601 [16]. Western blotting (Fig. 2a) and luciferase reporter assay (Fig. 2b) showed that FOXO1 expression and transcriptional activity were increased, respectively, in stably FOXO1 AAA expressing cells compared with vector control cells. Under normal culture conditions, FOXO1
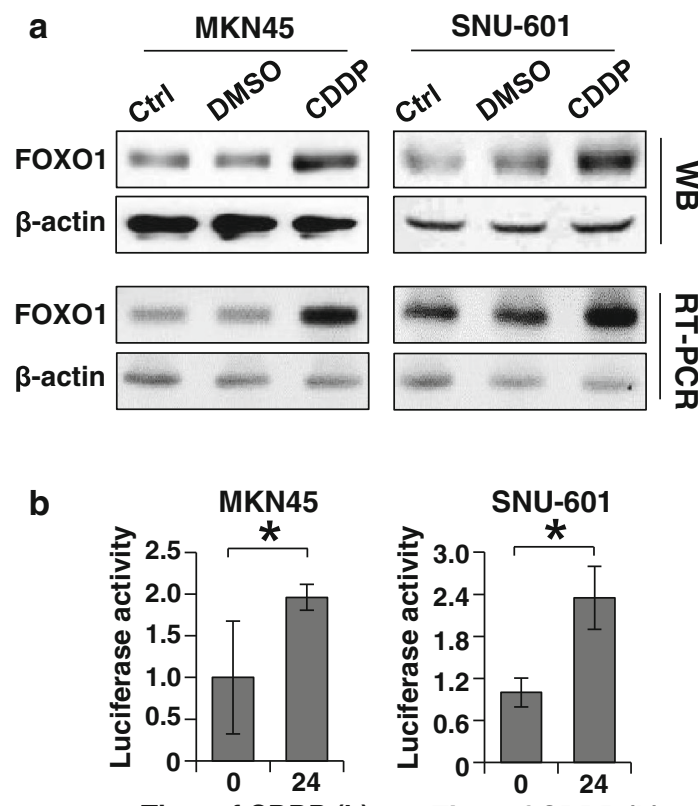

Time of CDDP (h)

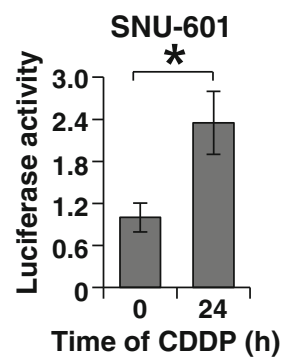

Fig. 1 Effects of cisplatin (CDDP) on FOXO1 expression and activity in gastric cancer cell lines. MKN45 and SNU-601 cells grown in $10-\mathrm{cm}$ plates were treated with either DMSO $(0.02 \%)$ or CDDP $(3 \mu \mathrm{g} / \mathrm{ml})$ for $24 \mathrm{~h}$. a Protein and mRNA expressions of FOXO1 were determined by Western blotting (WB) and semiquantitative reverse transcription-polymerase chain reaction (SQ RT-PCR) (RT-PCR), respectively. $\beta$-Actin protein and mRNA were used as internal controls. Ctrl, untreated control. b FOXO1 transcriptional activity was determined by luciferase reporter assay and normalized by $\beta$-galactosidase activity. Each bar represents the mean \pm standard deviation. ${ }^{*} P<0.05$ versus untreated cells overexpression had no effect on the cell growth of the gastric cancer cells (Fig. 2c). By contrast, treatment with CDDP $(0-3 \mu \mathrm{g} / \mathrm{ml})$ for $24 \mathrm{~h}$ showed that FOXO1 overexpression increased resistance to CDDP (Fig. 2d).

To further confirm our results, as already mentioned, gene silencing by RNA interference was used. FOXO1 expression (Fig. 3a) and activity (Fig. 3b) were suppressed by FOXO1 shRNA expression in both cell lines. There was no difference in growth rates between gastric cancer cells

a

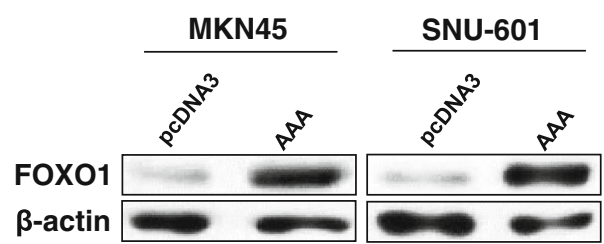

b
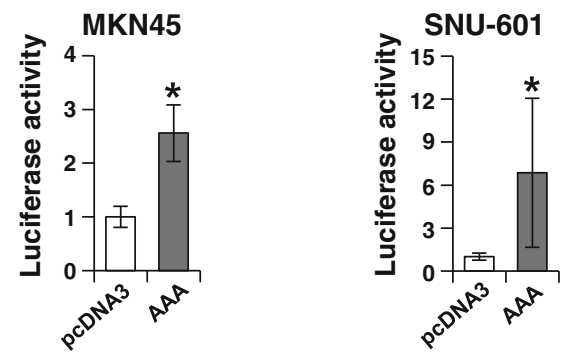

C
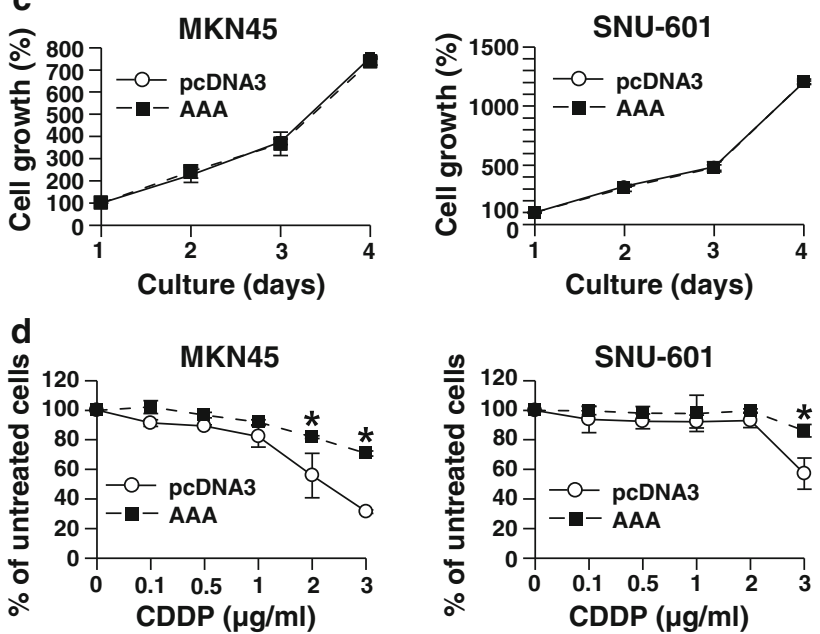

Fig. 2 Effects of FOXO1 AAA mutant expression on cisplatin (CDDP)-induced cytotoxicity in gastric cancer cell lines MKN45 and SNU-601. Cells were transfected with either an empty pcDNA3 vector (pcDNA3) or FOXO1 AAA mutant vector (AAA). a Expression levels of FOXO1 and $\beta$-actin proteins were confirmed by Western blotting. b FOXO1 transcriptional activity was determined by luciferase reporter assay and was normalized by $\beta$-galatosidase activity. Each bar represents mean \pm standard deviation. c Cell growth was analyzed using crystal violet assay on indicated times, and absorbance was measured. Values represent means \pm standard deviation. d Cells were treated with $\operatorname{CDDP}(0-3 \mu \mathrm{g} / \mathrm{ml})$ for $24 \mathrm{~h}$. Cell survivals represent the mean percentage survivals compared to untreated cells; values represent mean \pm standard deviation. $* P<0.05$ versus vector control cells 
Fig. 3 Effects of FOXO1 silencing on cisplatin (CDDP)induced cytotoxicity in gastric cancer cell lines MKN45 and SNU-601. Cells were infected with a lentivirus containing a construct encoding either FOXO1 shRNA (shFOXO1) or nontargeting shRNA (shCtrl). a, b FOXO1 silencing was confirmed by Western blotting

(a) and luciferase reporter assay (b). c, d The effects of FOXO1 downregulation on the cell growth (c) and CDDP cytotoxicity (d) were analyzed as described in Fig. 2.

${ }^{*} P<0.05$ versus shCtrl cells. e Cells were treated with CDDP $(1 \mu \mathrm{g} / \mathrm{ml}$ ) for $24 \mathrm{~h}$. Poly(ADP ribose)polymerase (PARP) cleavage was determined by Western blotting. f $4^{\prime}, 6^{\prime}$ Diamidino-2-phenylindole staining and fluorescence microscopy show morphological change in gastric cancer cells. $\times 400$
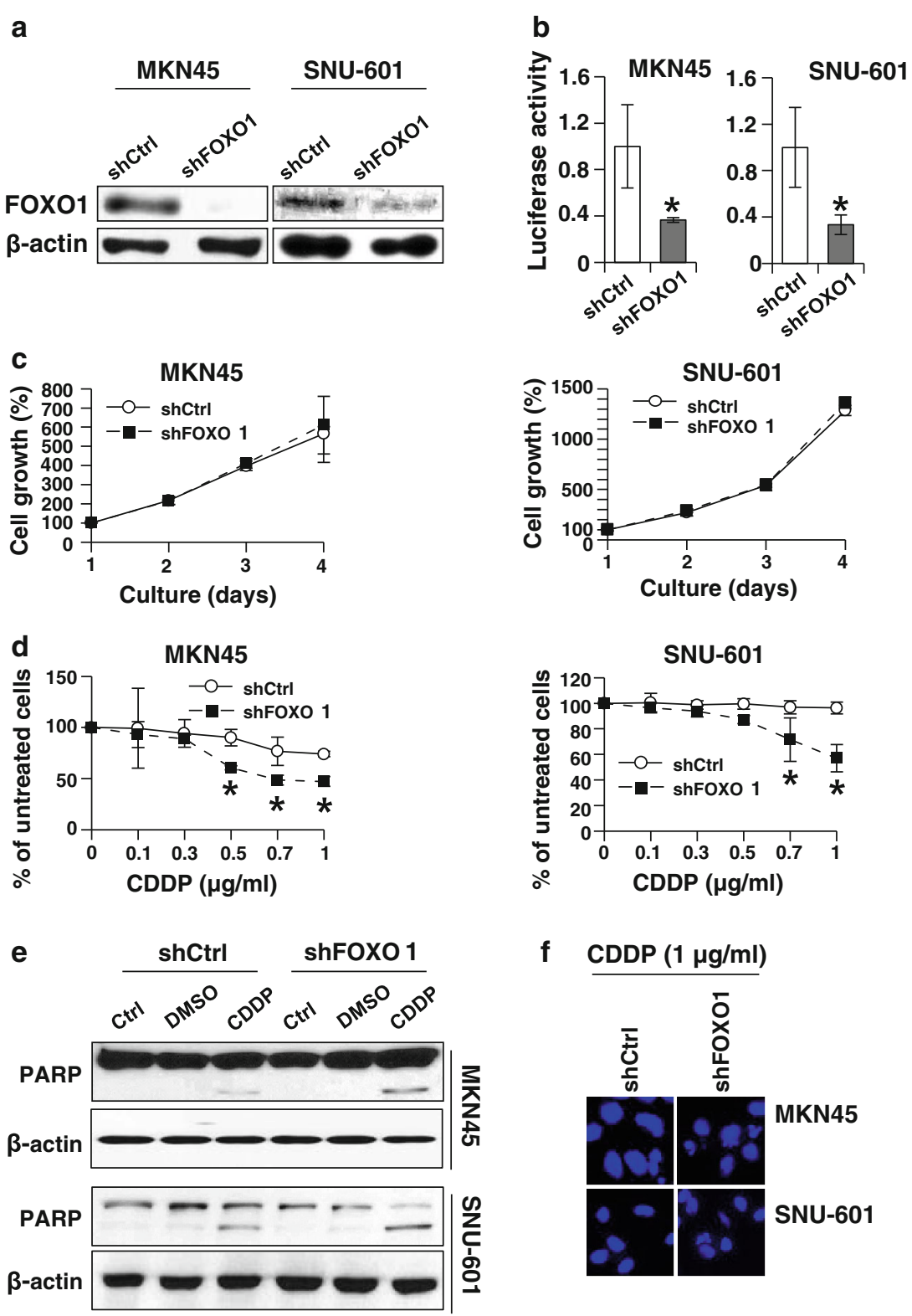

expressing nontargeting shRNA or FOXO1 shRNA (Fig. 3c). To confirm the effect of FOXO1 on resistance to CDDP, cells were treated with CDDP $(0-1 \mu \mathrm{g} / \mathrm{ml})$ for 24 h. Figure $3 d$ shows that FOXO1 silencing increased CDDP cytotoxicity in gastric cancer cells compared to cells without FOXO1 silencing. To examine whether CDDPinduced cell death occurs via apoptosis, we performed Western blotting for PARP (a caspase substrate) cleavage. After CDDP $(1 \mu \mathrm{g} / \mathrm{ml})$ treatment for $24 \mathrm{~h}$, cleaved PARP was increased in FOXO1 shRNA-expressing cells compared to control shRNA-expressing cells (Fig. 3e). The presence of apoptosis was confirmed by DAPI staining, which showed more frequent peripheral chromatin condensation and nuclear fragmentation in FOXO1 shRNA cells than in control shRNA cells (Fig. 3f). Taken together, these results indicate that FOXO1 protects gastric cancer cells to CDDP-induced cell death.

FOXO1 activation enhances PI3K/Akt activity

Recently, it has been reported that Akt overexpression decreases the chemosensitivity of gastric cancer cells to CDDP in vitro and in vivo [12]. Although FOXO1 is a well-known downstream substrate of Akt, FOXO1 was shown to enhance Akt phosphorylation in hepatocytes [17]. Thus, we investigated whether FOXO1-induced CDDP resistance attributes to Akt activation in gastric cancer cells. First, we hypothesized that FOXO1 may regulate the expression of PI3K, an upstream molecule of Akt. Western blotting showed that FOXO1 activaiton and silencing 

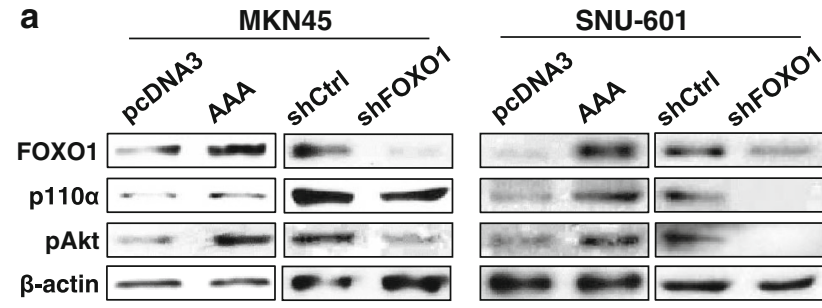

b
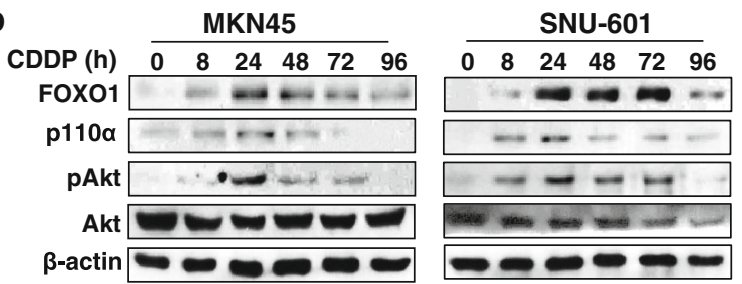

Fig. 4 Effect of FOXO1 activation on the expressions of p110 $\alpha$ and pAkt in gastric cancer cell lines MKN45 and SNU-601. Protein expressions were analyzed by Western blotting using specific antibodies against FOXO1, p110 $\alpha$, pAkt, Akt, and $\beta$-actin. a FOXO1 overexpression was induced by transfection with either empty pcDNA3 vector or FOXO1 AAA mutant vector (AAA), and FOXO1 silencing by infection with a lentiviral FOXO1 shRNA (shFOXO1) or nontargeting shRNA (shCtrl). b Cells were treated with $1 \mu \mathrm{g} / \mathrm{ml}$ cisplatin (CDDP). Protein lysates were prepared at the times indicated

directly increased and decreased the expressions of $\mathrm{p} 110 \alpha$, the class I PI3K catalytic subunit, and pAkt, the active form of Akt, respectively (Fig. 4a). In addition, CDDP-induced FOXO1 expression was accompanied by the expressions of p110 $\alpha$ and pAkt (Fig. 4b).

FOXO1 enhances CDDP resistance through PI3K/Akt pathway

To confirm whether the positive correlation between FOXO1 and CDDP resistance is mediated by Akt, we increased constitutive FOXO1 activation by transfection of FOXO1 AAA mutant gene into gastric cancer cell lines and then assessed the effects of $20 \mu \mathrm{M}$ LY294002 treatment on CDDP-induced cytotoxicity. Western blotting showed that gastric cancer cells expressing FOXO1 AAA mutant gene showed higher pAkt expression than vector control cells, but LY294002 treatment for 24 h effectively blocked pAkt expression (Fig. 5a). Cytotoxicity assay showed that FOXO1 AAA cells treated with LY294002 showed higher CDDP cytotoxicity, which suggests that Akt inhibition enables gastric cancer cells to resume the CDDP sensitivity suppressed by FOXO1 overexpression (Fig. 5b).

\section{Discussion}

Because FOXO transcription factors are critical mediators of apoptosis in cytotoxicity-inducing drugs, their involvement in the development of drug resistance is an important issue in cancer therapy [6]. However, FOXO proteins possess diverse functions, which partly seemed conflicting, according to drug and cell type [5]. To the best of our knowledge, this is the first study to demonstrate the correlation between FOXO1 and chemoresistance in gastric cancer cells.

We have previously shown that phosphorylated FOXO1 is overexpressed in gastric cancer specimens and that FOXO1 inactivation is related to better prognosis of gastric cancer patients [18]. Although FOXO proteins, especially FOXO1 and FOXO3, have been reported to be related to chemoresistance in various cancer cells [8-10, 19-21], their involvement in chemoresistance of gastric cancer cells has not been reported. In the present study, we used two gastric cancer cell lines, MKN45 and SNU-601, with constitutive FOXO1 expression and found that CDDP treatment increased the levels of FOXO1 protein expression and activation. Because CDDP treatment also increased FOXO1 mRNA expression, CDDP-induced FOXO1 transcription seems to contribute to the accumulation of FOXO1 protein. These results suggest that FOXO1 might impart the phenotype of CDDP resistance in gastric cancer cells.

Gene modulation is a powerful method for analyzing gene function [22]. Here, we confirmed the function of FOXO1 on CDDP resistance in two gastric cancer cell lines by modulation of FOXO1 activation using two different approaches (expression of FOXO1 AAA mutant gene and FOXO1 shRNA). We found that constitutive activation of FOXO1 increased the CDDP resistance in gastric cancer cells, whereas cell growth was not affected. In contrast, FOXO1 silencing with shRNA-enhanced CDDP-induced cytotoxicity accompanied by apoptotic characteristics, including cleaved PARP expression and fragmented nuclei. Taken together, these observations clearly demonstrated that FOXO1 is protective to CDDPinduced cytotoxicity in gastric cancer cells. Thus, blockage of FOXO1 could be an effective approach to improve the anti-cancer efficacy of CDDP in gastric cancer patients.

It is generally accepted that Akt is a critical survival signal, which is involved in cancer development and progression and chemoresistance [11]. Although cancer cells acquire resistance to anti-cancer agents through Akt, either constitutive or induced by anti-cancer drugs, the molecular mechanisms underlying anti-cancer drug-induced Akt activation are not well elucidated. In gastric cancer, we have observed that pAkt is expressed in $78 \%$ of gastric cancer specimens [23]. As a recent study showed that Akt overexpression decreases the chemosensitivity of gastric cancer cells to CDDP in vitro and in vivo [12], we investigated whether Akt is related to the FOXO1 regulation of CDDP cytotoxicity in gastric cancer cells. In the present study, constitutive activation and silencing of 
a
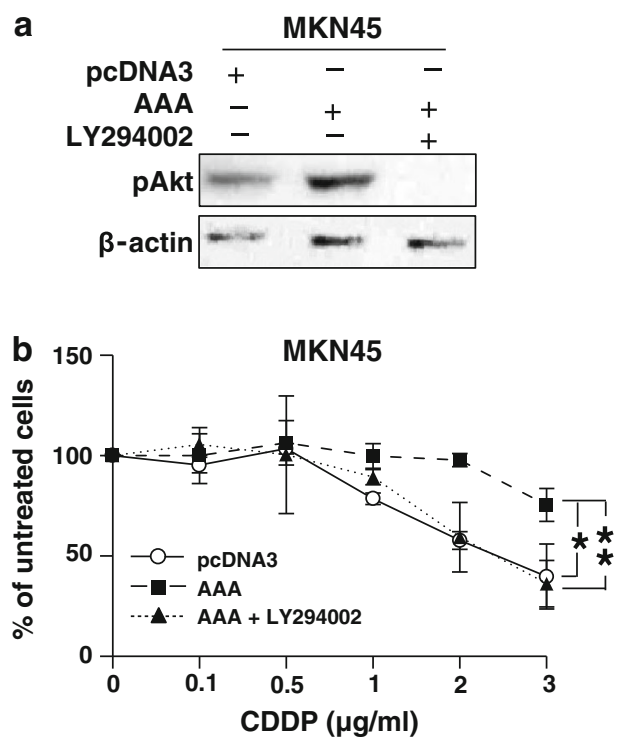

Fig. 5 Effect of PI3K inhibitor LY294002 on pAkt expression and cisplatin (CDDP) resistance induced by FOXO1 overexpression. MKN45 and SNU-601 cells were transfected with an empty pcDNA3 vector (pcDNA3) or a vector containing FOXO1 AAA mutant gene (AAA). a Cells were treated with $20 \mu \mathrm{M}$ LY294002 for $24 \mathrm{~h}$, and

FOXO1 increased and decreased the p110 $\alpha$ and pAkt expressions in gastric cancer cells, respectively, suggesting that FOXO1 activates PI3K/Akt signaling. Time-course analysis demonstrated that CDDP-induced FOXO1 expression was accompanied by concomitant increases in $\mathrm{p} 110 \alpha$ and pAkt expression. In addition, we found that treatment of FOXO1 AAA mutant gene-expressing gastric cancer cells with the PI3K/Akt inhibitor LY294002 resumed the CDDP cytotoxicity suppressed by FOXO1 overexpression. Thus, FOXO1 and Akt seem to have similar effects on CDDP resistance in gastric cancer cells. Our results agree with those of Hui et al. [19], which showed FOXO3-induced PI3K/Akt activation in response to doxorubicin treatment of chronic myelogenous leukemia cells. However, our observations contrast with those of Gao et al. [10], which demonstrated that FOXO1 enhanced CDDP cytotoxicity in ovarian cancer cells. These discrepancies may, at least in part, be explained by a previous suggestion by Paik et al. [24] that FOXO-regulated genes are different significantly between cell types. Indeed, FOXO1 induced multidrug resistance expression in breast cancer cells [25], whereas it upregulated the expressions of proapoptotic Bim and TRADD in ovarian cancer cells [10].

In conclusion, our results suggest that concurrent blocking of FOXO1 and Akt pathways is an effective strategy for improving the anti-cancer efficacy of CDDP. Thus, our results provide important clues for the efficient CDDP-design for future studies on gastric cancer treatment. Studies on the association between FOXO1 and
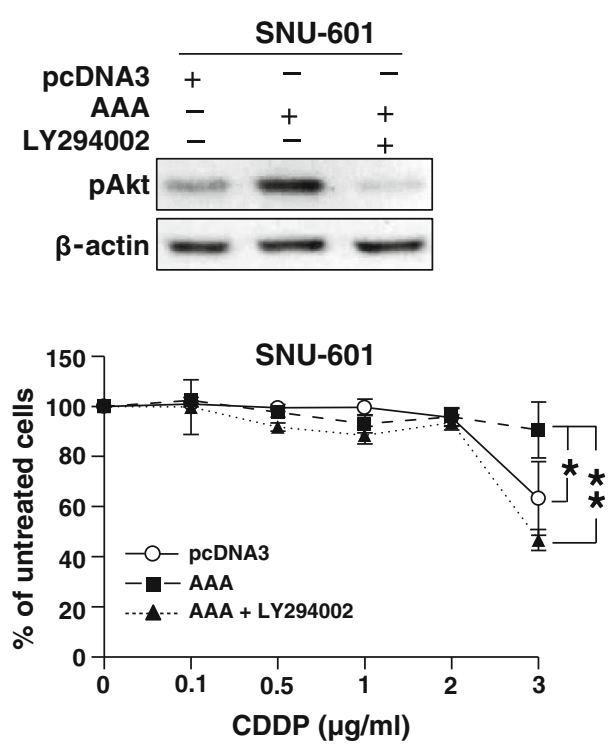

Western blotting was performed with an anti-pAkt antibody. b Cells were treated with CDDP $(0-3 \mu \mathrm{g} / \mathrm{ml})$ in the presence or absence of $20 \mu \mathrm{M}$ LY294002 for $24 \mathrm{~h}$. Cell survival represents mean percentage survivals compared to untreated cells; values represent mean \pm standard deviation

chemosensitivity in gastric cancer cells using human gastric cancer specimens with animal models are warranted to verify the usefulness of this strategy.

Acknowledgments This work was supported by grant no 04-20130570 from the SNUH Research Fund and National Research Foundation of Korea (NRF) Grant funded by the Korean Government (2010-0024702).

\section{References}

1. Crew KD, Neugut AI. Epidemiology of gastric cancer. World J Gastroenterol. 2006;12:354-62.

2. Koizumi W, Narahara H, Hara T, Takagane A, Akiya T, Takagi $\mathrm{M}$, et al. S-1 plus cisplatin versus S-1 alone for first-line treatment of advanced gastric cancer (SPIRITS trial): a phase III trial. Lancet Oncol. 2008;9:215-21.

3. Tanaka M, Kataoka H, Yano S, Ohi H, Kawamoto K, Shibahara T, et al. Anti-cancer effects of newly developed chemotherapeutic agent, glycoconjugated palladium (II) complex, against cisplatinresistant gastric cancer cells. BMC Cancer. 2013;13:237-45.

4. Maiese K, Chong ZZ, Shang YC, Hou J. Clever cancer strategies with FoxO transcription factors. Cell Cycle. 2008;7:3829-39.

5. Lei H, Quelle FW. FOXO transcription factors enforce cell cycle checkpoints and promote survival of hematopoietic cells after DNA damage. Mol Cancer Res. 2009;7:1294-303.

6. Goto T, Takano M. Transcriptional role of FOXO1 in drug resistance through antioxidant defense systems. Adv Exp Med Biol. 2009;665:171-9.

7. Zhang X, Tang N, Hadden TJ, Rishi AK. Akt, FoxO and regulation of apoptosis. Biochim Biophys Acta. 2011;1813:1978-86.

8. Oh WK, Cho KB, Hien TT, Kim TH, Kim HS, Dao TT, et al. Amurensin G, a potent natural SIRT1 inhibitor, rescues doxorubicin responsiveness via down-regulation of multidrug resistance 1. Mol Pharmacol. 2010;78:855-64. 
9. Goto T, Takano M, Hirata J, Tsuda H. The involvement of FOXO1 in cytotoxic stress and drug-resistance induced by paclitaxel in ovarian cancers. Br J Cancer. 2008;98:1068-75.

10. Gao J, Yang X, Yin P, Hu W, Liao H, Miao Z, et al. The involvement of FoxO in cell survival and chemosensitivity mediated by Mirk/Dyrk1B in ovarian cancer. Int $\mathrm{J}$ Oncol. 2012;40:1203-9.

11. Shimamura H, Terada Y, Okado T, Tanaka H, Inoshita S, Sasaki $\mathrm{S}$. The PI3-kinase-Akt pathway promotes mesangial cell survival and inhibits apoptosis in vitro via NF-kappa B and Bad. J Am Soc Nephrol. 2003; 14:1427-34.

12. Zhang LL, Zhang J, Shen L, Xu XM, Yu HG. Overexpression of AKT decreases the chemosensitivity of gastric cancer cells to cisplatin in vitro and in vivo. Mol Med Rep. 2013;7:1387-90.

13. Fukunaga K, Ishigami T, Kawano T. Transcriptional regulation of neuronal genes and its effect on neural functions: expression and function of forkhead transcription factors in neurons. J Pharmacol Sci. 2005;98:205-11.

14. Kim WH, Schnaper HW, Nomizu M, Yamada Y, Kleinman HK. Apoptosis in human fibrosarcoma cells is induced by a multimeric synthetic Tyr-Ile-Gly-Ser-Arg (YIGSR)-containing polypeptide from laminin. Cancer Res. 1994;54:5005-10.

15. Wang X, Gorospe M, Huang Y, Holbrook NJ. p27Kip1 overexpression causes apoptotic death of mammalian cells. Oncogene. 1997;15:2991-7.

16. Nakamura N, Ramaswamy S, Vazquez F, Signoretti S, Loda M, Sellers WR. Forkhead transcription factors are critical effectors of cell death and cell cycle arrest downstream of PTEN. Mol Cell Biol. 2000;20:8969-82.

17. Matsumoto M, Han S, Kitamura T, Accili D. Dual role of transcription factor FoxO1 in controlling hepatic insulin sensitivity and lipid metabolism. J Clin Invest. 2006;116:2464-72.
18. Kim JH, Kim MK, Lee HE, Cho SJ, Cho YJ, Lee BL, et al. Constitutive phosphorylation of the FOXO1A transcription factor as a prognostic variable in gastric cancer. Mod Pathol. 2007;20:835-42.

19. Hui RC, Gomes AR, Constantinidou D, Costa JR, Karadedou CT, Fernandez de Mattos S, et al. The forkhead transcription factor FOXO3a increases phosphoinositide-3 kinase/Akt activity in drug-resistant leukemic cells through induction of PIK3CA expression. Mol Cell Biol. 2008;28:5886-98.

20. Kong W, He L, Coppola M, Guo J, Esposito NN, Coppola D, et al. MicroRNA-155 regulates cell survival, growth, and chemosensitivity by targeting FOXO3a in breast cancer. J Biol Chem. 2010;285:17869-79.

21. Tezil T, Bodur C, Kutuk O, Basaga H. IKK- $\beta$ mediates chemoresistance by sequestering FOXO3; a critical factor for cell survival and death. Cell Signal. 2012;24:1361-8.

22. Brummelkamp TR, Bernards R, Agami R. Stable suppression of tumorigenicity by virus-mediated RNA interference. Cancer Cell. 2002;2:243-7.

23. Nam SY, Lee HS, Jung GA, Choi J, Cho SJ, Kim MK, et al. Akt/ PKB activation in gastric carcinomas correlates with clinicopathologic variables and prognosis. APMIS. 2003;111:1105-13.

24. Paik JH, Kollipara R, Chu G, Ji H, Xiao Y, Ding Z, et al. FoxOs are lineage-restricted redundant tumor suppressors and regulate endothelial cell homeostasis. Cell. 2007;128:309-23.

25. Han CY, Cho KB, Choi HS, Han HK, Kang KW. Role of FoxO1 activation in MDR1 expression in adriamycin-resistant breast cancer cells. Carcinogenesis (Oxf). 2008;29:1837-44. 